Comment. Math. Helv. 74 (1999) 248-279

(C) 1999 Birkhäuser Verlag, Basel

$0010-2571 / 99 / 020248-32 \$ 1.50+0.20 / 0$

Commentarii Mathematici Helvetici

\title{
The singly periodic genus-one helicoid
}

\author{
David Hoffman, Hermann Karcher and Fusheng Wei
}

\begin{abstract}
We prove the existence of a complete, embedded, singly periodic minimal surface, whose quotient by vertical translations has genus one and two ends. The existence of this surface was announced in our paper in Bulletin of the AMS, 29(1):77-84, 1993. Its ends in the quotient are asymptotic to one full turn of the helicoid, and, like the helicoid, it contains a vertical line. Modulo vertical translations, it has two parallel horizontal lines crossing the vertical axis. The nontrivial symmetries of the surface, modulo vertical translations, consist of: $180^{\circ}$-rotation about the vertical line; $180^{\circ}$ rotation about the horizontal lines (the same symmetry); and their composition.
\end{abstract}

Mathematics Subject Classification (1991). 53A10, 53C42, 49F10.

Keywords. Minimal surface, embedded, elliptic functions, Riemann surfaces.

\section{Introduction}

In this paper, we prove the existence of a complete, embedded, singly periodic minimal surface, whose quotient by vertical translations has genus one and two ends. The existence of this surface was announced in [4] and its significance discussed in [5]. Its ends in the quotient are asymptotic to one full turn of the helicoid, and, like the helicoid, it contains a vertical line. Modulo vertical translations, it has two parallel horizontal lines crossing the vertical axis. The nontrivial symmetries of the surface, modulo vertical translations, consist of: $180^{\circ}$ rotation about the vertical line; $180^{\circ}$ rotation about the horizontal lines (the same symmetry); and their composition - a $180^{\circ}$ rotation about a line, orthogonal to the lines on the surface, and passing through their point of intersection. This line meets the

Hoffman was supported by research grant DE-FG03-95ER25250 of the Applied Mathematical Science subprogram of the Office of Energy Research, U.S. Department of Energy. Hoffman and Wei were supported by research grant DMS-95-96201 of the National Science Foundation, Division of Mathematical Sciences. Research at MSRI is supported in part by NSF grant DMS90-22140 


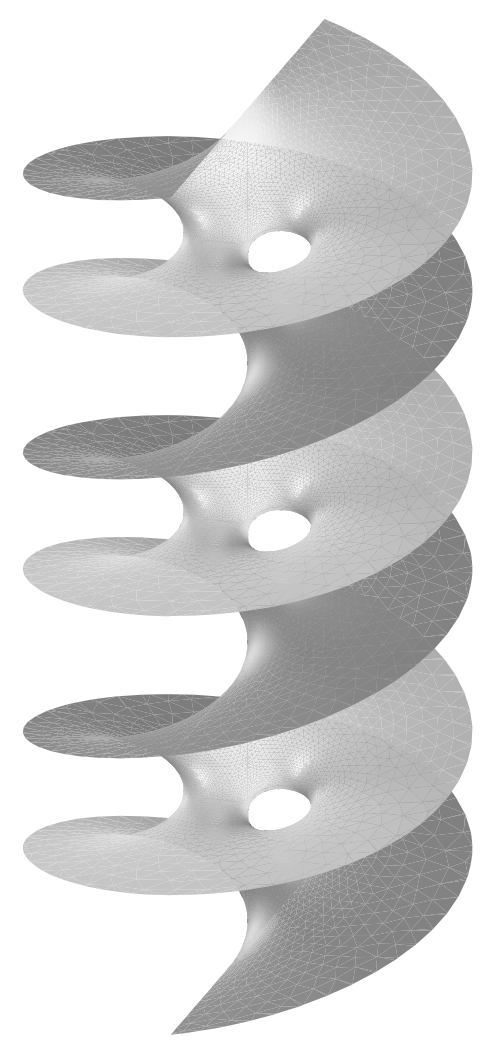

Figure 1.

The periodic genus-one helicoid.

surface orthogonally and is referred to as a normal symmetry line.

The description of the qualitative properties of the surface in the paragraph above is sufficient to determine a two-parameter family of Weierstrass data (1.7) that must contain the Weierstrass data for any surface with these properties - if it exists. One parameter controls the conformal type of the quotient, in this case a rhombic torus. The other can be considered as controlling the placement of the punctures corresponding to the ends. This is worked out in Section 1 and presented in Theorem 1.

The proof of existence of the singly periodic genus-one helicoid consists of showing that the period problem $((1.8),(1.9))$ is solvable. This is done in Theorem 2 of Section 2. In Theorem 3 of Section 3, we prove that the surface is embedded by decomposing a fundamental domain into disjoint graphs. As usual, the existence and embeddedness proofs are independent. We do not use any special properties of the parameters that kill the periods. In fact we show that any singly periodic 
(by translations) minimal surface that, in the quotient, is asymptotic to the helicoid (1.1) and contains a vertical axis and two horizontal parallel lines must be embedded.

Other than the helicoid itself, this example was the first embedded minimal surface ever found that is asymptotic to the helicoid. It was one of the important steps in the discovery and construction of the non-periodic genus-one helicoid, whose existence is proved in [5]. We hope that a complete understanding of this periodic surface will be helpful in giving a non-computational proof, which is not complete as of this writing, of the embeddedness of the genus-one helicoid.

We might have discovered this periodic surface earlier, had we been looking for it at the time. In 1989, the first two authors realized that a construction of Fischer and Koch [1, 2] could be modified to produce singly periodic, embedded minimal surfaces with multiple helicoidal ends. The Fischer-Koch triply periodic surface is formed of pieces congruent to the solution to the disk-type Plateau Problem for the boundary in Figure 2.

The surface extends, by $180^{\circ}$ rotation about its boundary line segments, to a triply periodic embedded surface. Our modification consisted of two simple steps. First, we realized that the length of the sides marked $a_{i}$ could be increased without limit, producing an embedded minimal graph over a strip. This extends to an embedded, singly periodic surface with six flat ends of Scherk-type. Second, we observed that the fundamental piece could be modified by rotating the horizontal sides $a_{1}$ in Figure 2 by a fixed angle, say $\theta$, with respect to the sides $a_{2}$. Each fixed $\theta$ produces a fundamental embedded piece that extends, by $180^{\circ}$ rotation about the line segments on the boundary, to an embedded minimal surface, asymptotic to three coaxial helicoids, and invariant under a vertical screw motion of the form

$$
p \rightarrow e^{2 i \theta} p+(0,0,8 b)
$$

Its Weierstrass representation is suggestive of the surface to which we now turn our attention.

\section{Determination of the Weierstrass Representation}

The helicoid can be described by the data

$$
g=z, d h=\frac{i d z}{z}
$$

on $\mathcal{S}=\mathbb{C}-\{0\}$ in the Weierstrass representation ([3])

$$
X(p)=X\left(p_{0}\right)+\operatorname{Re} \int_{p_{0}}^{p} \Phi, \quad \Phi=\left(\frac{1}{2}\left(g^{-1}-g\right), \frac{i}{2}\left(g^{-1}+g\right), 1\right) d h .
$$



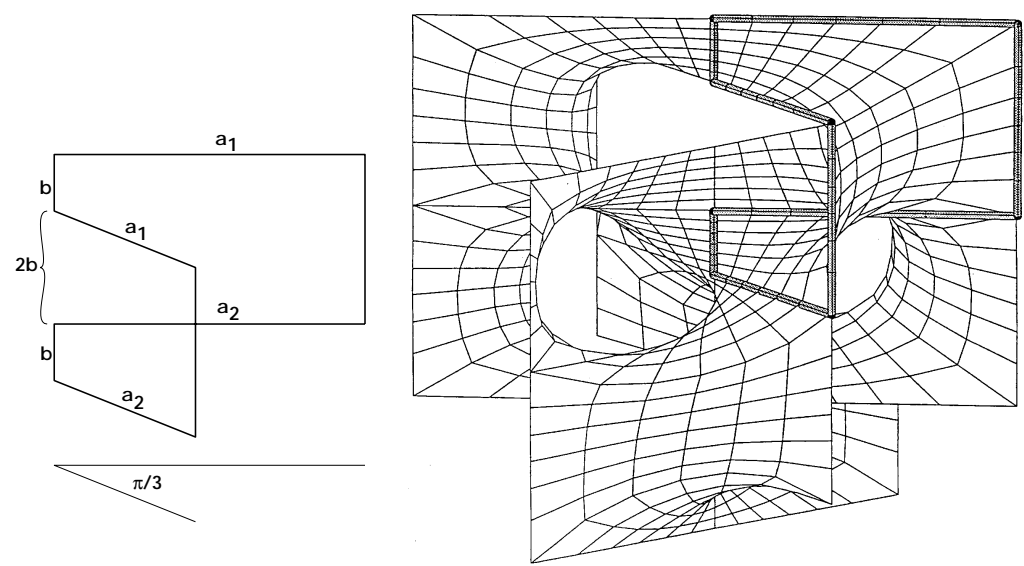

Figure 2.

Above left: The boundary of a fundamental building block of one of the Fischer-Koch triply periodic surfaces. Above right: Six copies of the fundamental building block, which together form a fundamental domain of the triply periodic surface. The surface is computed using the discrete minimal surface ideas and subsequent code of Pinkall and Polthier [7]. The computation was carried out by Bernd Oberknapp. The original computation of the surface was done by Ortwin Wohlrab; it was his pictures that suggested to the first two authors the singly periodic surfaces described in the text, one of which is illustrated below. Below: A singly periodic minimal surface, invariant under a vertical screw-motion and asymptotic to three coaxial helicoids. It was suggested by the Fischer-Koch surface, above right.

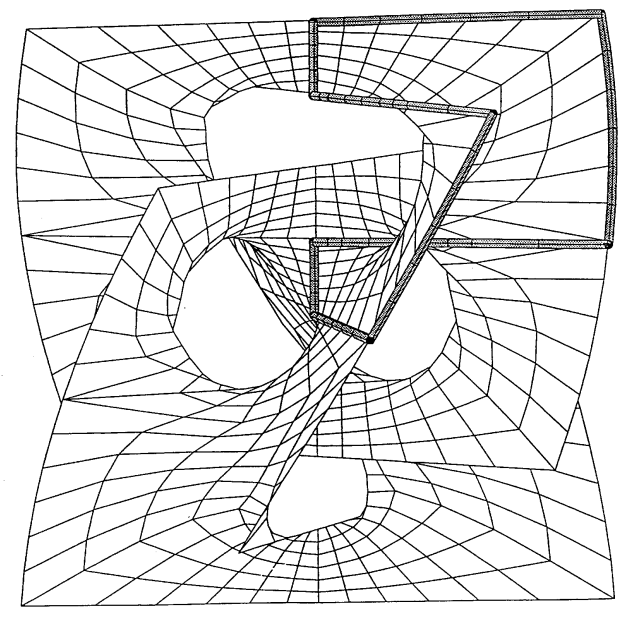

The integration produces a conformal minimal immersion, but has a period equal 
to $(0,0, \pm 2 \pi)$ on any closed curve $\gamma$ homotopic to $|z|=1$. Thus the immersion in (1.2) with data (1.1) is multivalued, and its image, the helicoid, is invariant under a vertical translation produced by the period of (1.2) on the cycle $|z|=1$.

The minimal surface we wish to construct is singly periodic, has a helicoidaltype end and, modulo translations, will have genus equal to one. From general results [6] and our assumptions about the geometry and topology of the surface in the Introduction, we know that (if it exists) it will have a Weierstrass representation on a twice-punctured torus and that $g$ and $d h$ will extend meromorphically to the compact torus. ${ }^{1}$ Our goal is to determine the Weierstrass representation from this information and from the symmetry we assume the surface to have.

\subsection{The lines on the surface}

We assume that, like the helicoid, our surface contains a single vertical line andagain like the helicoid - the symmetry of $180^{\circ}$ rotation about this line fixes this line and no other points. This implies that the torus is a rhombic torus, which we denote by $T^{2}$.

We assume in addition that the surface contains, modulo translations, two parallel horizontal lines, each meeting the vertical line in a single point. Rotation of $180^{\circ}$ about either of these lines fixes both lines in the quotient. Because each of these lines connects the two punctures, such a rotation leaves the punctures corresponding to the ends unmoved . Each line diverges to both ends, forcing the fixedpoint set on the torus of this $180^{\circ}$ rotation to be a single closed symmetry curve that contains both end-punctures. This curve must cross the curve corresponding to the vertical axis in two points. The two punctures separate the symmetry line into two components corresponding to the two horizontal lines. These two points must be symmetrically placed with respect to $180^{\circ}$ rotation about the vertical line.

We choose a fundamental domain for our torus so that the lines on the surface correspond to the diagonals of a rhombus. Because we will use our development of elliptic functions in [5], these diagonals are placed to make $\mathrm{a} \pm 45^{\circ}$ angle with the real axis in $\mathbb{C}$. See Figure 3. We think of them as the horizontal and the vertical diagonal, according to the lines on the surface in space, which are their images. To avoid possible confusion with lines in the $\mathbb{C}$ and in $\mathbb{R}^{3}$, we will refer to them as the v-diagonal and the h-diagonal. Without loss of generality, we may assume that the h-diagonal is the one making a $45^{\circ}$ angle with the real axis.

Wherever possible, we will deal only with elliptic functions, avoiding any notational reference to the complex variable in $\mathbb{C}$, which is not well defined on the torus. However we will have need of $d u$ - the differential of a complex variable $u$ - which projects to the torus and is free of poles and zeros.

Rotation by $180^{\circ}$ about a point on the surface where the straight lines meet is

\footnotetext{
1 The helicoid data in (1.1) is defined on a twice-punctured sphere and it clearly extends meromorphically.
} 
a rotation about the line determined by the normal to the surface at that point, we refer to it as a normal symmetry. On the quotient torus it is an orientationpreserving involution: $180^{\circ}$ rotation about the center of the torus. We denote this rotation by $r_{P}$. It fixes four points that are on a half-period lattice. We may choose as a fundamental domain for the torus a rhombus whose center is at one of these points and whose vertex is another such point. These two points are the intersection of the diagonals of the rhombus. The other two fixed points of the normal symmetry both lie at the same height as one or the other of the fixed points that lie on the vertical axis. We assume, without loss of generality, that they lie at the same height as the fixed point corresponding to the center of the rhombus.

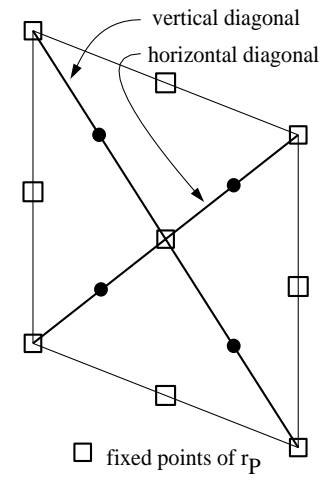

- fixed points of $\mathrm{r}_{\mathrm{D}}$

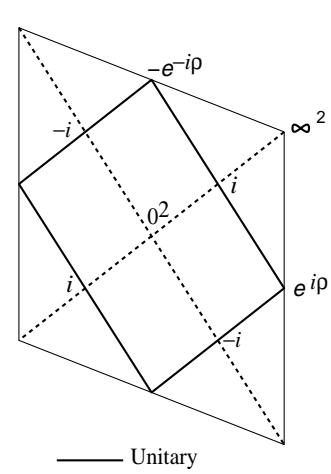

.......- Imaginary

Figure 3.

Left: Fixed points of $r_{D}$ and $r_{P}$ The diagonals are labeled vertical and horizontal here because they are the preimages of the vertical and horizontal lines on the surface. In the text, they are referred to as the $\mathrm{v}$ - and h-diagonals. Right: Values of the elliptic function $z$.

\subsection{Elliptic functions associated to our construction}

Euler's formula implies that $T^{2} / r_{P}$ is the sphere. The quotient map $T^{2} \rightarrow T^{2} / r_{P}$ is meromorphic, has degree two, and is branched at the half-period points. We want to use this map to describe the torus analytically. To do this it is necessary to turn it into a function by identifying three points of the sphere $T^{2} / r_{P}$ with points in the complex plane. The motivation for our choice is to make the symmetries of the torus induce simple symmetries of $\mathbb{C} \cup\{\infty\}$. This is explained in detail in [5].

Denote by $O$ (resp. $O^{\prime}$ ) the center point (resp. the vertex) of $T^{2}$. The choice we make is to have $0 \in \mathbb{C}$ equal the projection of $O$, and $\infty$ equal the projection of $O^{\prime}$ (since these points are fixed by $r_{P}$, these values are branch values) and $+i$ equal 
the projection of the midpoint of the h-diagonal. This determines a degree-two elliptic function, which we denote by $z$. See Figure 3 .

Let $r_{D}$ denote $180^{\circ}$ rotation around a midpoint of a diagonal between $O$ and $O^{\prime}$. It fixes all four such midpoints. Observe that $r_{D}$ interchanges $O$ and $O^{\prime}$ in $T^{2}$, where $z=0, \infty$ respectively, and fixes a point where $z=i$. This implies that $z \circ r_{D}=-1 / z$. Thus $z= \pm i$ at the fixed points of $r_{D}$. Since $z \circ r_{P}=z$, by definition, and the degree of $z$ is two, the values of $z= \pm i$ at the fixed points of $r_{D}$ must be as in Figure 3 .

Let $\mu$ be reflection in a diagonal of $T^{2}$. Then $\overline{z \circ \mu}=-z$. To see this, first observe that both functions in question are meromorphic on $T^{2}$. Their quotient has no zero or poles, and takes on the value 1 at a fixed point of $r_{D}$. This implies that $z$ has imaginary values on the diagonals. Similarly, let $\nu$ denote reflection in the line parallel to a diagonal and passing through a fixed point of $r_{D}$. Then $\bar{z} \circ \nu=1 / z$. Thus $|z|=1$ on this line.

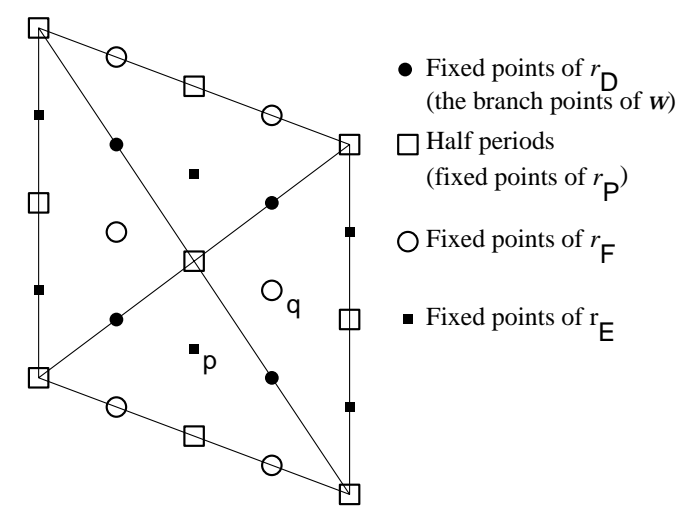

Figure 4.

The fixed point sets of the four involutions of the torus. Each involution is $180^{\circ}$ rotation about its fixed points.

In particular, the values of $z$ at the two half-period points other than $O, O^{\prime} \in T^{2}$ are of the form $e^{i \rho}$ and $-e^{-i \rho}$, because $r_{D}$ interchanges these points and $z \circ r_{D}=$ $-1 / z$.

In fact, $z$ is a geometric normalization of the Weierstrass $\wp$-function. It satisfies the differential equation

$$
\left(\frac{\frac{d z}{d u}}{z}\right)^{2}=-\frac{2}{\cos \rho}\left(z-\frac{1}{z}-2 i \sin \rho\right)
$$

The value of $\rho$ characterizes the rhombic torus as can be seen explicitly in (1.3) above and Lemma 1 iii) below. 
Before returning to the determination of the Weierstrass data, we will construct another elliptic function intimately related to the minimal surface. This time we will use the involution $r_{D}$, which fixes four points on the diagonals. The projection $T^{2} \rightarrow T^{2} / r_{D}$ is a degree-two meromorphic map that we will turn into an elliptic function by specifying its values at three points of $S^{2}=T^{2} / r_{D}$. At the projection of $O \in T$ we specify the value 0 . At the projection of one of the half periods (not $O^{\prime}$ ) we specify the value $\infty$. Because $w \circ r_{D}=w$ by definition, this determines $w$ at the half-period points. (See Figure 5.) We now determine $w$ by specifying its value to be 1 at the point $q$ indicated in Figure 4 .

The following Lemma contains information we will need about $w$ in our discussion of the surface. In the proof of the lemma and in discussions that follow, we will need to distinguish between reflection in the h-diagonal and the v-diagonal. Let $\mu$ denote reflection in the h-diagonal and write $\mu_{v e r t}$ for reflection in the $\mathrm{v}$-diagonal.

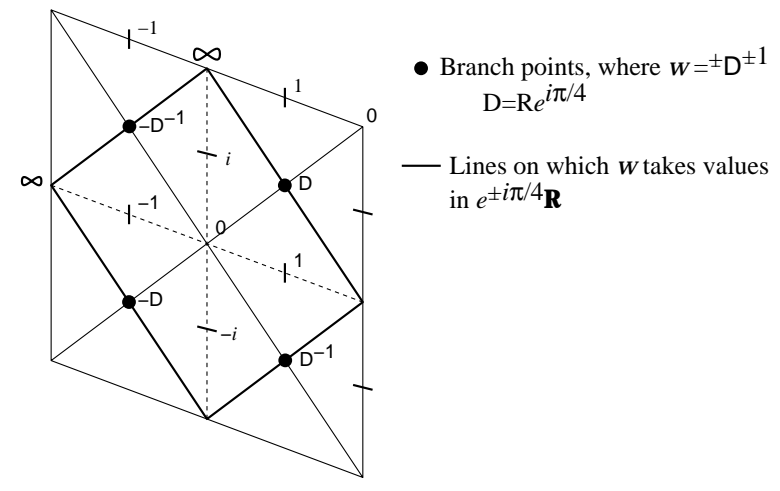

Figure 5.

The values of the elliptic function $w$.

Lemma 1. The degree-two elliptic function $w$ on a rhombic torus has values as indicated in Figure 5:

(i) Along the diagonals and along the lines through the branch points, $w$ takes on values in $e^{ \pm i \pi / 4} \mathbb{R}$. In particular, its branch values are of the form $\pm R e^{ \pm i \pi / 4}$, the constant $R>0$ being determined by the choice of rhombus;

(ii) For a fixed rhombic torus, the branch values of $w$ are related to the branch value $e^{i \rho}$ of $z$ by $R^{2}=\cot (\pi / 4-\rho / 2)$;

(iii) The rhombic torus is determined by the algebraic relation

$$
(2 \cos \rho) w^{-2}=-\left(z-z^{-1}-2 i \sin \rho\right) .
$$

Proof of Lemma 1. We will prove (i) here. Statement (iii) implies Statement 
(ii) by explicit calculation. Both are proved in [5], where there is a fuller discussion of Jacobian elliptic functions.

By definition, $w \circ r_{D}=w$, so the placement of the points where $w=1$ is determined. Let $r_{F}$ denote $180^{\circ}$-rotation around $q$, an involution whose other fixed points are marked by $\circ$ in Figure 4. The meromorphic functions $w \circ r_{F}$ and $w^{-1}$ agree at $q$ and have the same zeros and poles. Hence $w \circ r_{F}=w^{-1}$. We already knew $w=1$ at two of the fixed points of $r_{F}$; hence $w=-1$ at the other two. In the same manner $w \circ r_{P}$ and $w$ have the same zeros and poles and, noting the values of $w$ at the fixed points of $r_{F}$, one sees that $w \circ r_{P}=-w$. Similarly, $r_{E}$ is $180^{\circ}$ rotation about $p$ (see Figure 4), and $w \circ r_{E}=-w^{-1}$. This implies that $w= \pm i$ at the fixed points of $r_{E}$. The signs are determined by the fact that $w$ is orientation preserving.

The meromorphic function $\overline{w \circ \mu_{v e r t}}$ has the same zeros and poles as $w$. Evaluation at $q$ gives the relation $\overline{w \circ \mu_{\text {vert }}}=i w$. This means that $w$ takes on values in $e^{-i \pi / 4} \mathbb{R}$ on the $\mathrm{v}$-diagonal. If $\mu$ is reflection in the $\mathrm{h}$-diagonal $\overline{w \circ \mu}=-i w$ and therefore $w$ takes on values in $e^{i \pi / 4} \mathbb{R}$ on this line. Similarly, reflection $\nu$ (resp. $\hat{\nu}$ ) in the lines parallel to the diagonals of the rhombus and passing through the other two fixed points of $r_{P}$ satisfies $\overline{w \circ \nu}=i w($ resp. $\overline{w \circ \hat{\nu}}=-i w)$, so $w$ takes values in $e^{i \pi / 4} \mathbb{R}\left(\right.$ resp. $\left.e^{-i \pi / 4} \mathbb{R}\right)$ on these lines.

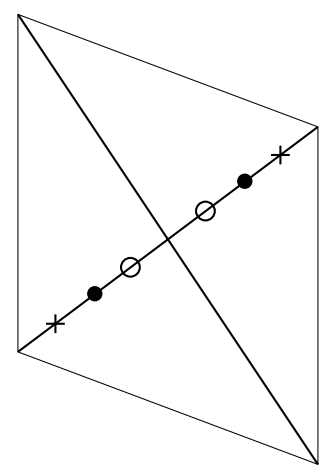

Figure 6 .

A - marks the branch points of $g$ on the h-diagonal, and a $\circ$ marks the points on the surface where the Gauss map is vertical, i.e. $g$ is zero or infinity. An $\mathrm{x}$ marks the punctures. For this illustration, we are assuming that $\lambda<1$; for $\lambda>1$, the punctures (x) and vertical points $(0)$ are interchanged.

\subsection{The Gauss map in terms of $w$}

From [6], we know that the total curvature of our desired quotient surface is $-2 \pi(\chi(M)-W(M))$, where $\chi(M)$ is the Euler characteristic of the quotient surface 
$M$, and $W(M)$ is the total winding number of the ends of $M$. In our case, $M$ is a torus $T^{2}$ punctured twice, so $\chi(M)=-2$. Both ends are asymptotic to a single full turn of the helicoid, so each end contributes 1 to $W(M)$. Hence the total curvature is $-8 \pi$ and the degree of $g$ is 2 .

Because we want a helicoidal end on the surface, as well as a vertical line that corresponds to the axis of the helicoid, we see from (1.1) that $g=0$ at one puncture and $g=\infty$ at the other. There must be one other zero and one other pole. Because rotation about a vertical (or horizontal) line in $\mathbb{R}^{3}$ will leave zeros and poles of $g$ unchanged (or interchange them), the other zero and pole of $g$ must lie on the symmetry lines; otherwise there would be too many zeros and poles. On the v-diagonal, $g$ must be unitary, so the zeros and poles are on the h-diagonal.

As is the case for any degree-two elliptic function, the branch points of $g$ are symmetrically placed with respect to the zeros and poles.(See [5].) Because the end-punctures are also on the h-diagonal, two branch points are on this diagonal, too. The four branch points of $g$ form a set that is invariant under symmetries of the surface. In particular, the branch-point set is invariant under $r_{P}$ (induced by the normal symmetry). This, together with the fact that two branch points of $g$ are on the h-diagonal, implies that the branch points of $g$ are the quarter-points of the diagonals; these are exactly the branch points of $w$. Thus $g$ and $w$ differ by a Möbius transformation.

At a puncture, $w= \pm r e^{i \pi / 4}$ for some $r>0$, a consequence of Lemma 1(ii) in Section 1.2. Because $g$ is unitary at $O \in T^{2}$, it follows that

$$
g=e^{i \theta} \frac{w-r e^{i \pi / 4}}{w+r e^{i \pi / 4}}
$$

and, after a rotation about a vertical axis, if necessary, we may assume that $\theta=0$, i.e.

$$
g=\frac{w-r e^{i \pi / 4}}{w+r e^{i \pi / 4}}
$$

\subsection{The complex height differential in terms of $z$}

We can easily determine the differential $d h$, which is holomorphic on the punctured surface. From (1.2) (or see [3]) the metric on the surface is given by $d s=(|g|+$ $\left.|g|^{-1}\right)|d h|$. Because we require $d s$ to be everywhere nonzero (for regularity), $d h$ has simple zeros at the two points where the Gauss map is vertical on the surface. Thus it has only two poles, which must be located at the punctures. In particular, $d h \circ r_{P}=d h$.

In Section 1.1 we set up the parameter domain so that the h-diagonal would be mapped into a horizontal line in $R^{3}$. This forces $d h$ to be imaginary on the h-diagonal; i.e. $x_{3}=\operatorname{Re} \int d h$ must be constant on this line.

Just as $w$ was well adapted to $g, z$ is a good match for $d h$. Recall (or see Figure 3 ) that $z$ is imaginary on both diagonals and, by definition, $z \circ r_{P}=z$. We 
define $\lambda$ by the requirement that $z=i / \lambda$ at the punctures. Since $z \circ r_{D}=-1 / z$, $z=i \lambda$ at the vertical points of $g$ on the h-diagonal. On this diagonal, $z$ takes values on the nonnegative imaginary axis and therefore $\lambda>0$. Also, $\lambda \neq 1$ because this $g$ is not branched at a helicoidal end. The function

$$
\frac{z-i \lambda}{z-i \lambda^{-1}}
$$

has the same poles and zeros as $d h$ and is real on the diagonals.

We can express $d h$ in terms of the standard pole-and-zero-free holomorphic form $d u$, described in Section 1.1. Under our assumption that the h-diagonal makes a $45^{\circ}$ angle with the real axis, $e^{i \pi / 4} d u$ is imaginary on this diagonal. Hence, we may assume without loss of generality that

$$
d h=e^{i \pi / 4} \frac{z-i \lambda}{z-i \lambda^{-1}} d u
$$

because we are free to scale the surface by a real constant.

Remark 1. Using Lemma 1 (iii) we can relate $\lambda$ to $\mathrm{r}$ :

$$
\lambda+\lambda^{-1}=2\left(\sin \rho+(\cos \rho) / r^{2}\right) .
$$

\subsection{The Ansatz for the periodic genus-one helicoid}
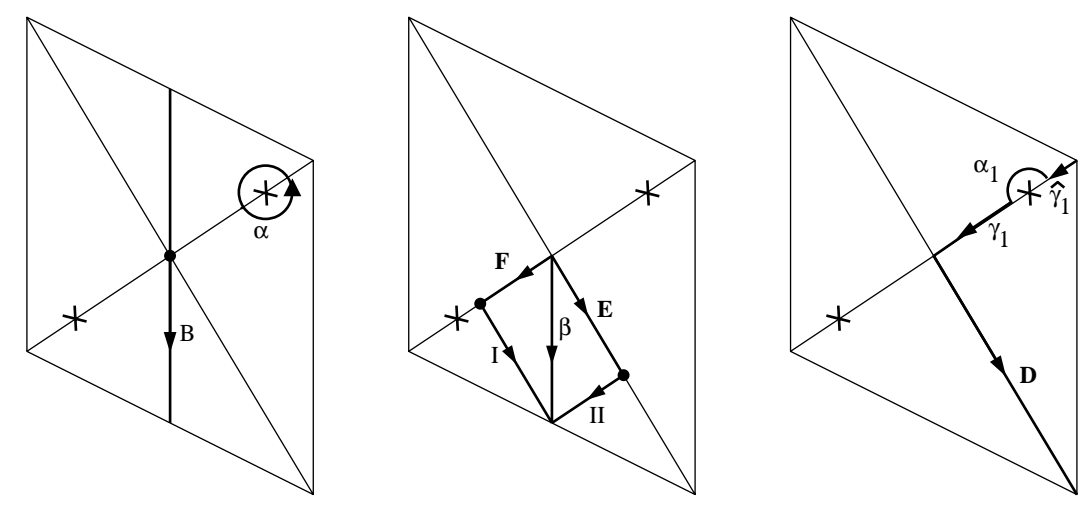

Figure 7 .

Left: Two cycles that, together with their reflections across the v-diagonal, generate the homology of the punctured torus. Center: Paths used to derive the period conditions (1.8) and (1.9). Right: A cycle homologous to $B$. 
Our geometric description of the surface has led to an explicit two-parameter family of Weierstrass data: For $\rho \in(-\pi / 2, \pi / 2)$ and $\lambda \in(0,1) \cup(1, \infty)$,

$$
g=\frac{w-r e^{i \pi / 4}}{w+r e^{i \pi / 4}}, \quad d h=e^{i \pi / 4} \frac{z-i \lambda}{z-i \lambda^{-1}} d u
$$

on the rhombic torus

$$
(2 \cos \rho) w^{-2}=-\left(z-z^{-1}-2 i \sin \rho\right),
$$

punctured at the two points where $z=i \lambda^{-1}$.

Theorem 1. For every $\rho \in(-\pi / 2, \pi / 2)$ and every $\lambda \in(0,1) \cup(1, \infty)$, the Weierstrass data, (1.7) - defined on the rhombic torus determined by $\rho$, with punctures where $z=i \lambda^{-1}$ on the h-diagonal-produces in (1.2) a multivalued, regular, complete minimal immersion of the punctured torus. It has the following properties:

(i) Reflection in the v-diagonal (resp. h-diagonal) of the torus induces an isometry of the minimal surface corresponding to $180^{\circ}$ rotation about a vertical line (resp. horizontal line) in $\mathbb{R}^{3}$;

(ii) The immersion (1.2) is singly periodic if and only if $\lambda \in(0,1), \rho \in(0, \pi / 2)$ and

$$
\begin{aligned}
\operatorname{Re} \int_{I} d h & =0 \\
\operatorname{Re} \int_{I I} i\left(g^{-1}+g\right) d h & =0
\end{aligned}
$$

where the paths I and II are as indicated in Figure 7. The period ${ }^{2}$ is a vertical vector of the form $(0,0, T)$, where $T$ is given in (1.16) and

$$
T=\int_{\text {"v-diagonal" }} d h= \pm 2 \pi i\left(\text { Residue }_{z=i \lambda^{-1}} d h\right) .
$$

Conversely, any regular, complete, periodic, minimal surface containing a vertical line, whose quotient by vertical translations has genus one, contains two parallel horizontal lines and has two helicoidal ends, is representable with Weierstass data of the form (1.7) with $\lambda \in(0,1)$ and $\rho \in(0, \pi / 2)$.

Proof. The "converse" part of the Theorem is clear - from the development in previous sections, any such surface must be represented by the data as given in

2 Since the surface can be scaled by multiplying dh by a nonzero real constant, the value of $T$ has no geometric significance. For the representation we use here, $T$ is given explicitly in (1.16) as a function of $\rho$ and $\lambda$. 
(1.7) with punctures at the two points where $z=i \lambda^{-1}$. The statements that $\lambda \in(0,1)$ and that $\rho \in(0, \pi / 2)$ will be proved below.

We begin the proof of (i) and (ii) by considering the one-form $d h$ in (1.7), which was constructed to have its zeros precisely at the two points where $g=0$ or $g=\infty$ on the punctured torus. Hence the metric

$$
d s=\frac{1}{2}\left(|g|+|g|^{-1}\right)|d h|
$$

is regular. It is complete because at the punctures, where $g=0$ or $g=\infty, d h$ has a simple pole.

Since the h- (resp. v-) diagonal makes an angle of $45^{\circ}$ (resp. $\left.-45^{\circ}\right)$ with the positive real axis, $\mu^{*} d u=i \overline{d u}$ (resp. $\mu_{v e r t}^{*} d u=-i \overline{d u}$ ). From Section 1.2, we know that $z \circ \mu_{\text {vert }}=z \circ \mu=-\bar{z}$. It follows from the definition of $d h$ in (1.7) that

$$
\mu^{*} d h=-\overline{d h} \text { and } \mu_{v e r t}^{*} d h=\overline{d h} .
$$

From Section 1.2 we have $w \circ \mu=i \bar{w}$ (resp. $w \circ \mu_{\text {vert }}=-i \bar{w}$ ). A computation using the definition of $g$ in (1.7) gives

$$
g \circ \mu=\bar{g} \text { and } g \circ \mu_{\text {vert }}=1 / \bar{g} .
$$

The Weierstrass representation (1.2) is $X(p)=X\left(p_{0}\right)+\operatorname{Re} \int_{p_{0}}^{p} \Phi$,

$$
\Phi:=\left(\phi_{1}, \phi_{2}, d h\right)=\left(\frac{1}{2}\left(g^{-1}-g\right), \frac{i}{2}\left(g^{-1}+g\right), 1\right) d h .
$$

From (1.11), (1.12) and the relation $r_{P}=\mu \circ \mu_{\text {vert }}$ we have:

$$
\begin{aligned}
\mu^{*} \Phi & =\overline{\left(-\phi_{1}, \phi_{2},-d h\right)} \\
\mu_{\text {vert }}^{*} \Phi & =\overline{\left(-\phi_{1},-\phi_{2}, d h\right)} \\
r_{P}{ }^{*} \Phi & =\left(\phi_{1},-\phi_{2},-\phi_{3}\right) .
\end{aligned}
$$

Statement (i) of the theorem follows immediately from the Weierstrass representation and these relations: writing $X=:\left(x_{1}, x_{2}, x_{3}\right)$, and assuming, without loss of generality, that $p_{0}$ is at the center of the rhombus and and $X\left(p_{0}\right)=(0,0,0)$, then

$$
\begin{aligned}
X \circ \mu(p) & =\left(-x_{1}, x_{2},-x_{3}\right)(p) \\
X \circ \mu_{\text {vert }}(p) & =\left(-x_{1},-x_{2}, x_{3}\right)(p) \\
X \circ r_{P}(p) & =\left(x_{1},-x_{2},-x_{3}\right)(p)
\end{aligned}
$$

We now address the period problem and begin by computing the period at a puncture corresponding to an end. At the punctures where $z=i \lambda^{-1}, \phi_{3}=d h$ 
has a pole. Note that when $z=i \lambda^{-1}, w= \pm r e^{i \pi / 4}$, so $g=0$ or $\infty$ there. Because $g^{-1} d h$ and $g d h$ both have their only pole at one of the puncturesand therefore no residue $\phi_{1}$ and $\phi_{2}$ have no residues there. Let $\alpha$ be a simple closed curve in the homotopy class of a puncture. (See Figure 7.) Then $\int_{\alpha} \Phi=2 \pi i\left(0,0\right.$, Residue $\left._{z=i \lambda^{-1}} d h\right)$. From (1.7) we have

$$
\begin{aligned}
d h=e^{i \pi / 4} \frac{z-i \lambda}{z-i \lambda^{-1}} d u & =e^{i \pi / 4} \frac{z-i \lambda}{z-i \lambda^{-1}}\left(\frac{z^{\prime}}{z}\right)^{-1} \frac{d z}{z} \\
& =e^{i \pi / 4} \frac{z-i \lambda}{z}\left(\frac{-2}{\cos \rho}\left(z-z^{-1}-2 i \sin \rho\right)\right)^{-1 / 2} \frac{d z}{z-i \lambda^{-1}}
\end{aligned}
$$

Hence

$$
\begin{aligned}
\operatorname{Residue}_{z=i \lambda^{-1}} d h & =e^{i \pi / 4} \frac{\lambda^{-1}-\lambda}{\lambda^{-1}}\left(\frac{-4 i}{\cos \rho}\left(\frac{\lambda+\lambda^{-1}}{2}-\sin \rho\right)\right)^{-1 / 2} \\
& =-i \frac{\sqrt{\cos \rho}}{2}\left(1-\lambda^{2}\right)\left(\frac{\lambda+\lambda^{-1}}{2}-\sin \rho\right)^{-1 / 2}
\end{aligned}
$$

and

$$
\operatorname{Period}_{\alpha} X:=\operatorname{Re} \int_{\alpha} \Phi=(0,0, \pm T)
$$

where

$$
T:=\pi \sqrt{\cos \rho}\left(1-\lambda^{2}\right)\left(\frac{\lambda+\lambda^{-1}}{2}-\sin \rho\right)^{-1 / 2} \neq 0 .
$$

Let $\alpha_{1}$ and $\beta$ be as in Figure 7. Note that we may write $\alpha=\alpha_{1}-\mu \alpha_{1}$. We note for use below that because (from (1.13)) $\mu^{*} d h=-\overline{d h}$ and $\mu^{*} \phi_{1}=-\overline{\phi_{1}}$,

$$
\operatorname{Re} \int_{\alpha_{1}} \phi_{1}=\operatorname{Re} \int_{-\mu \alpha_{1}} \phi_{1} \text { and } \operatorname{Re} \int_{\alpha_{1}} d h=\operatorname{Re} \int_{-\mu \alpha_{1}} d h
$$

It follows from this and (1.15) that

$$
\operatorname{Re} \int_{\alpha_{1}} \phi_{1}=\operatorname{Re} \int_{-\mu \alpha_{1}} \phi_{1}=0 \text { and } \operatorname{Re} \int_{\alpha_{1}} d h=\operatorname{Re} \int_{-\mu \alpha_{1}} d h= \pm T / 2
$$

At the end of Section 1.1 we chose, without loss of generality, to represent the surface so that the image of the center point of the torus was at the same height as the two off-axis fixed points of the normal symmetry, i.e. these are the fixed points of $r_{P}$ (see Figure 3). Thus we must require

$$
\operatorname{Re} \int_{\beta} d h=0
$$


where $\beta$ is the curve in Figure 7. By (1.14), it follows that $\operatorname{Re} \int_{\mu \beta} d h=0$, so both off-axis fixed points are at the same level, thus satisfying a necessary condition from Section 1.1.

Any branch of $X$ maps the v-diagonal to a vertical line segment and, since $g(O)=-1$, the h-diagonal is mapped to a line parallel to the $x_{2}$-axis. Hence the image under any branch of $X$ of the off-axis fixed points of $r_{P}$ must lie on a line, passing through $X(O)$, which is parallel to the $x_{1}$-axis. That is

$$
\operatorname{Re} \int_{\beta} \phi_{2}=\operatorname{Re} \int_{\beta} \frac{i}{2}\left(g^{-1}+g\right) d h=0 .
$$

Hence $\operatorname{Re} \int_{\beta} \Phi=\left(\operatorname{Re} \int_{\beta} \phi_{1}, 0,0\right)$.

For $\hat{\beta}:=r_{P} \beta$, we have from (1.13) that $\operatorname{Re} \int_{\hat{\beta}} \Phi=\left(\operatorname{Re} \int_{\beta} \phi_{1}, 0,0\right)$. This means that on the closed cycle $B:=+\beta-\hat{\beta}$,

$$
\operatorname{Period}_{B} X=\operatorname{Re} \int_{B} \Phi=(0,0,0)
$$

The four closed curves $\left\{B, \mu B, \alpha, \mu_{v e r t} \alpha\right\}$ form a spanning set for the homology of the punctured torus. By (1.13) it follows that $\operatorname{Period}_{B} X=(0,0,0)$ if and only if Period $_{\mu B} X=(0,0,0)$. This means that the necessary conditions (1.18) and (1.19) are sufficient to insure that $\mathrm{X}$ has no real period on either $B$ or $\mu B$. By (1.15) and (1.16), $\mathrm{X}$ always has a nonzero vertical period on $\alpha$ and - by symmetry, using (1.13) - on $\mu_{\text {vert }} \alpha$. Therefore, (1.18) and (1.19) are sufficient to guarantee that $X$ is singly periodic with vertical period $(0,0, T)$.

Before translating (1.18) and (1.19) into (1.8) and (1.9), we will show that (1.10) is valid. Observe that $B$ is homotopic to $D+\gamma_{1}+\alpha_{1}+\gamma_{2}$. (See Figure 7.) We know that $D$ is mapped into a vertical line line segment so $\operatorname{Re} \int_{\gamma_{1}+\alpha_{1}+\gamma_{2}} \Phi$ is also a vertical vector. The $\gamma_{i}$ lie on the horizontal diagonal and are mapped to horizontal line segments parallel to the $x_{2}$-axis. It follows from this and (1.17) that

$$
\operatorname{Re} \int_{D} \Phi=-\left(0,0, \operatorname{Re} \int_{\alpha_{1}} d h\right)=(0,0,-T / 2)
$$

This is equivalent to (1.10) in the statement of Theorem 1.

Equations (1.18) and (1.19) are the period conditions: necessary and sufficient conditions for the surface in question to be singly periodic. We wish to express them in a form more convenient for subsequent calculation, i.e. as equations (1.8) and (1.9). When $\lambda<1, \beta$ is homotopic to $F+I$ and to $E+I I$. See Figure 7 . Along $F$, we know that $x_{3}$ is constant. Thus (1.18) is equivalent to

$$
\operatorname{Re} \int_{I} d h=0
$$


which is (1.8). Similarly, $x_{2}$ is constant along $E$, so (1.19) is equivalent to

$$
\operatorname{Re} \int_{I I} \frac{i}{2}\left(g^{-1}+g\right) d h=0
$$

which is (1.9).

We now show that the period conditions cannot be satisfied when $\lambda>1$. On $E, z=-i t, 0 \leq t \leq 1$. We may use $z$ to parametrize $E$ by $t: z \circ E(t)=-i t$; $E(t)=f(t) e^{-i \pi / 4}$, where $f(t)$ is some positive increasing function. Then

$$
\begin{aligned}
d h(\dot{E}) & =e^{i \pi / 4} \frac{-i t-i \lambda}{-i t-i \lambda^{-1}} e^{-i \pi / 4} \dot{f}(t) \\
& =\frac{t+\lambda}{t+\lambda^{-1}} \dot{f}(t) .
\end{aligned}
$$

Hence

$$
a:=x_{3}(E(1))=\operatorname{Re} \int_{E} d h>0 .
$$

Along $I I$, which begins at $b:=E(1), z=-i e^{-i t}$, for $0 \leq t \leq \pi / 2+\rho$. Also, $z$ is branch-point-free along $I I$, so we may use $z$ to parametrize $I I$ by $t$ : $z \circ I I(t)=-i e^{-i t} ; I I(t)=b-f(t) e^{+i \pi / 4}$, where $f(t)$ is, again, some positive increasing function. Then

$$
\begin{aligned}
d h(\dot{I I}) & =e^{i \pi / 4} \frac{-i e^{-i t}-i \lambda}{-i e^{-i t}-i \lambda^{-1}} e^{i \pi / 4}(-\dot{f}(t)) \\
& =-i \dot{f}(t) \frac{e^{-i t}+\lambda}{e^{-i t}+\lambda^{-1}} \\
& =\left(\left(\lambda-\lambda^{-1}\right) \sin t+i(\ldots)\right) \frac{\dot{f}(t)}{\left|e^{-i t}+\lambda^{-1}\right|^{2}}
\end{aligned}
$$

Thus $\operatorname{Re} d h(\dot{I I})$ is strictly positive when $\lambda>1$. This means that $x_{3}(t)$ is strictly increasing. But since $a=x_{3}(b)>0$, this would imply that $\operatorname{Re} \int_{\beta} d h=\operatorname{Re} \int_{E+I I} d h>$ 0 , violating (1.18).

This completes the proof of Theorem 1, with the exception of showing that the period conditions cannot be solved for $\rho \leq 0$. This will be proved in Lemma 2 below.

Remark 2. We note for use in Section 3 that (1.21) implies that when $\lambda<1$, $x_{3} \circ I I(t)$ is strictly decreasing. 


\subsection{The period conditions in terms of definite integrals}

In this section we express the integrals in (1.8) and (1.9) in terms of explicit definite integrals. We will need this for the existence proof in Section 2 as well as for the proof of

Lemma 2. The period condition (1.9) is not solvable when $\rho \leq 0$.

Along paths I and II, where $z=e^{i \phi}$, we have

$$
\begin{aligned}
e^{i \pi / 4} d u & =e^{i \pi / 4} \frac{z}{z^{\prime}} \frac{d z}{z}=e^{i \pi / 4}\left(-\frac{2}{\cos \rho}\left(e^{i \phi}-e^{-i \phi}-2 i \sin \rho\right)\right)^{-1 / 2} i d \phi \\
& =\left\{\begin{array}{cl}
\frac{\sqrt{\cos \rho}}{2} \frac{d \phi}{\sqrt{\sin \phi-\sin \rho}} & \text { for } \rho<\phi<\pi / 2, \\
-i \frac{\sqrt{\cos \rho}}{2} \frac{d \phi}{\sqrt{\sin \rho-\sin \phi}} & \text { for }-\pi / 2<\phi<\rho
\end{array}\right.
\end{aligned}
$$

and

$$
\begin{aligned}
\frac{z-i \lambda}{z-i \lambda^{-1}} & =\frac{e^{i \phi}-i \lambda}{e^{i \phi}-i \lambda^{-1}} \frac{\lambda e^{-i \phi}+i}{\lambda e^{-i \phi}+i} \\
& =\lambda \frac{2-\left(\lambda+\lambda^{-1}\right) \sin \phi+i\left(\lambda^{-1}-\lambda\right) \cos \phi}{\lambda+\lambda^{-1}-2 \sin \phi}
\end{aligned}
$$

Let $\Lambda=\lambda+\lambda^{-1}$. Using (1.7) and the expressions above, we see that the first period condition (1.8) is equivalent to

$$
\int_{\rho}^{\frac{\pi}{2}} \frac{2-\Lambda \sin \phi}{\Lambda-2 \sin \phi} \frac{d \phi}{\sqrt{\sin \phi-\sin \rho}}=0 .
$$

To write the second period condition (1.9), we first compute

$$
\begin{aligned}
g+\frac{1}{g} & =\frac{w-r e^{i \pi / 4}}{w+r e^{i \pi / 4}}+\frac{w+r e^{i \pi / 4}}{w-r e^{i \pi / 4}}=2 \frac{w^{2}+i r^{2}}{w^{2}-i r^{2}} \\
& =2 \frac{\Lambda-4 \sin \rho+2 \sin \phi}{\Lambda-2 \sin \phi}
\end{aligned}
$$

The last equality follows from (1.6) and the definition of $\Lambda=\lambda+\lambda^{-1}$. Together with the expression for $d h$ computed above, this shows that condition (1.9) is equivalent to

$$
\int_{-\frac{\pi}{2}}^{\rho} \frac{\Lambda-4 \sin \rho+2 \sin \phi}{\Lambda-2 \sin \phi} \frac{2-\Lambda \sin \phi}{\Lambda-2 \sin \phi} \frac{d \phi}{\sqrt{\sin \rho-\sin \phi}}=0
$$

For $\rho \leq 0$, no factor of the integrand of (1.23) changes sign, so the second period condition is not solvable for $-\pi / 2<\rho \leq 0$. 


\section{Existence}

Theorem 2. There exists $\left(\rho_{0}, \lambda_{0}\right) \in(0, \pi / 2) \times(0,1)$ satisfying the period conditions (1.8) and (1.9).

Proof. In Section 1.6, we wrote the period conditions (1.8) and (1.9) as the definite integrals (1.22) and (1.23). We will work with these relations without reference to the geometry of their derivation:

$$
\begin{gathered}
\int_{\rho}^{\frac{\pi}{2}} \frac{2-\Lambda \sin \phi}{\Lambda-2 \sin \phi} \frac{d \phi}{\sqrt{\sin \phi-\sin \rho}}=0 \\
\int_{-\frac{\pi}{2}}^{\rho} \frac{\Lambda-4 \sin \rho+2 \sin \phi}{\Lambda-2 \sin \phi} \frac{2-\Lambda \sin \phi}{\Lambda-2 \sin \phi} \frac{d \phi}{\sqrt{\sin \rho-\sin \phi}}=0
\end{gathered}
$$

where $\Lambda=\lambda+\lambda^{-1}$. We need to find a pair $(\rho, \lambda)$ solving both of these equations simultaneously.

The proof goes as follows. Using the intermediate value theorem, we show in Section 2.1 that condition (2.1) can be solved for $(\rho, \lambda(\rho))$, where $\lambda(\rho)$ is a differentiable function of $\rho$ and $0<\rho<\pi / 2$. Then we show in Section 2.2 that the period integral in (2.2) changes sign along the graph of $\lambda=\lambda(\rho)$. By the intermediate value theorem again, there exists $\left(\rho_{0}, \lambda\left(\rho_{0}\right)\right)$ at which both $(2.1)$ and (2.2) hold.

\subsection{Solution of the first period integral as a function of $\rho$}

Consider, for $0<\rho<\pi / 2$, the first period integral in (2.1). Observe that the sign of the differentiable function

$$
F(\rho, \Lambda)=\int_{\rho}^{\frac{\pi}{2}} \frac{2-\Lambda \sin \phi}{\Lambda-2 \sin \phi} \frac{d \phi}{\sqrt{\sin \phi-\sin \rho}}
$$

can be determined from the sign of the integrand: positive for $\Lambda=2$; negative for $\Lambda \geq 2 / \sin \rho$. In particular,

$$
F(\rho, 2)>0 \quad \text { and } \quad F\left(\rho, \frac{2}{\sin \rho}\right)<0
$$

Also since the integrand is, for each fixed $\rho$, a decreasing function of $\Lambda$, the same is true of $F(\rho, \Lambda)$. Therefore we may define a differentiable function $\Lambda(\rho)$, for $0<\rho<\pi / 2$, by the condition

$$
F(\rho, \Lambda(\rho))=0
$$


By (2.3) we have

$$
2<\Lambda(\rho)<\frac{2}{\sin \rho}
$$

In order to estimate the second period integral along the graph of $\Lambda=\Lambda(\rho)$, we need to control $\Lambda(\rho)$.

Lemma 3. For $\rho \in(0, \pi / 2)$ we have

$$
2<\Lambda(\rho)<\min \left(\frac{2}{\sin \rho}, 8\right)
$$

For $\rho \in(\pi / 2-\epsilon, \pi / 2)$, where $\epsilon$ is sufficiently small, we have

$$
2<\Lambda(\rho)<2+(1-\sin \rho)
$$

Proof. Since we already have (2.3) and (2.4), it is enough to show that $F(\rho, 8)<0$ for $\rho \in(0, \pi / 2)$, and $F(\rho, 2+(1-\sin \rho))<0$ for $\rho$ near $\pi / 2$. Define, for $\Lambda<2 / \sin \rho$, $\phi_{\Lambda}$ to be the zero of the integrand of $F$, i.e.

$$
\sin \phi_{\Lambda}=\frac{2}{\Lambda}
$$

Then

$$
\begin{aligned}
F(\rho, \Lambda) & =\int_{\rho}^{\phi_{\Lambda}} \frac{2-\Lambda \sin \phi}{\Lambda-2 \sin \phi} \frac{d \phi}{\sqrt{\sin \phi-\sin \rho}} \\
& -\int_{\phi_{\Lambda}}^{\frac{\pi}{2}} \frac{\Lambda \sin \phi-2}{\Lambda-2 \sin \phi} \frac{d \phi}{\sqrt{\sin \phi-\sin \rho}}
\end{aligned}
$$

Both integrands are positive. Since $(2-\Lambda \sin \phi) /(\Lambda-2 \sin \phi)$ is decreasing in the interval $\left(\rho, \phi_{\Lambda}\right)$ and $(\cos \phi) /\left(\cos \phi_{\Lambda}\right) \geq 1$, we have

$$
\begin{aligned}
\int_{\rho}^{\phi_{\Lambda}} \frac{2-\Lambda \sin \phi}{\Lambda-2 \sin \phi} \frac{d \phi}{\sqrt{\sin \phi-\sin \rho}} & \leq \frac{2-\Lambda \sin \rho}{\Lambda-2 \sin \rho} \int_{\rho}^{\phi_{\Lambda}} \frac{\cos \phi / \cos \phi_{\Lambda}}{\sqrt{\sin \phi-\sin \rho}} d \phi \\
& =\frac{2-\Lambda \sin \rho}{\Lambda-2 \sin \rho} \frac{2 \sqrt{\sin \phi_{\Lambda}-\sin \rho}}{\cos \phi_{\Lambda}} \\
& =\frac{2-\Lambda \sin \rho}{\Lambda-2 \sin \rho} \cdot 2 \sqrt{\frac{2 / \Lambda-\sin \rho}{1-4 / \Lambda^{2}}}
\end{aligned}
$$


Turning our attention to the second integral in (2.5), we first estimate a term in the integrand. Let

$$
f(\phi):=\frac{\Lambda \sin \phi-2}{\Lambda-2 \sin \phi} .
$$

By the definition of $\phi_{\Lambda}, f\left(\phi_{\Lambda}\right)=0$ and clearly $f(\pi / 2)=1$. It is easy to check that $f^{\prime}(\phi)>0$ on $\left(\phi_{\Lambda}, \pi / 2\right)$, so $f(\phi)$ is increasing on this interval. Also one can compute that $f^{\prime}\left(\phi_{\Lambda}\right)=1 / \cos \left(\phi_{\Lambda}\right)>1 /\left(\pi / 2-\phi_{\Lambda}\right)$. Furthermore, $f^{\prime \prime}\left(\phi_{\Lambda}\right)>0$ and $f^{\prime \prime}(\phi)$ has only one zero on $\left(\phi_{\Lambda}, \pi / 2\right)$. The linear function

$$
l(\phi):=\frac{\phi-\phi_{\Lambda}}{\pi / 2-\phi_{\Lambda}}
$$

satisfies $l\left(\phi_{\Lambda}\right)=0=f\left(\phi_{\Lambda}\right)$ and $l(\pi / 2)=1=f(\pi / 2)$. Since $l^{\prime}(\phi)=1 /\left(\pi / 2-\phi_{\Lambda}\right)$, we have $f^{\prime}\left(\phi_{\Lambda}\right)>l^{\prime}\left(\phi_{\Lambda}\right)$. Because $l^{\prime \prime}(\phi)=0, f(\phi)>l(\phi)$ at least up to the first zero of $f^{\prime \prime}$. On the remaining subinterval, $f$ is concave, so it is above its secant, which is in turn above $l$. It follows that

$$
f(\phi)=\frac{\Lambda \sin \phi-2}{\Lambda-2 \sin \phi} \geq \frac{\phi-\phi_{\Lambda}}{\pi / 2-\phi_{\Lambda}}
$$

on $\left(\phi_{\Lambda}, \pi / 2\right)$.

We now can now estimate the integral in (2.5).

$$
\begin{aligned}
\int_{\phi_{\Lambda}}^{\frac{\pi}{2}} \frac{\Lambda \sin \phi-2}{\Lambda-2 \sin \phi} \frac{d \phi}{\sqrt{\sin \phi-\sin \rho}} & \geq \int_{\phi_{\Lambda}}^{\frac{\pi}{2}} \frac{\phi-\phi_{\Lambda}}{\pi / 2-\phi_{\Lambda}} \frac{d \phi}{\sqrt{1-\sin \rho}} \\
& =\frac{1}{2} \frac{\pi / 2-\phi_{\Lambda}}{\sqrt{1-\sin \rho}} \geq \frac{1}{2} \frac{\sin \left(\pi / 2-\phi_{\Lambda}\right)}{\sqrt{1-\sin \rho}} \\
& =\frac{1}{2} \frac{\cos \phi_{\Lambda}}{\sqrt{1-\sin \rho}}=\frac{1}{2} \frac{\sqrt{1-4 / \Lambda^{2}}}{\sqrt{1-\sin \rho}}
\end{aligned}
$$

These two integral estimates and (2.5) give

$$
F(\rho, \Lambda) \leq 2 \frac{2-\Lambda \sin \rho}{\Lambda-2 \sin \rho} \sqrt{\frac{2 / \Lambda-\sin \rho}{1-4 / \Lambda^{2}}}-\frac{1}{2} \sqrt{\frac{1-4 / \Lambda^{2}}{1-\sin \rho}} .
$$

The right-hand side consists of the difference between two terms. For $\rho \in(0, \pi / 2)$ the subtracted function is increasing in $\rho$ while the two terms in the first expression are decreasing functions of $\rho$. All together, the right-hand side is a decreasing function of $\rho \in(0, \pi / 2)$. At $\Lambda=8$ we have

$$
F(\rho, 8) \leq F(0,8) \leq 2 \cdot \frac{2}{8} \cdot \sqrt{\frac{2 / 8}{1-4 / 8^{2}}}-\frac{1}{2} \sqrt{1-\frac{4}{8^{2}}}<0 .
$$


This proves the first part of Lemma 3, that is,

$$
2<\Lambda(\rho)<\min \left(\frac{2}{\sin \rho}, 8\right)
$$

for $\rho \in(0, \pi / 2)$.

We now wish to estimate $\Lambda(\rho)$ for $\rho$ near $\pi / 2$. Let $\Lambda_{k}:=2+k(1-\sin \rho)$. Then

$$
F\left(\rho, \Lambda_{k}\right) \leq 2 \frac{(2-k \sin \rho)^{3 / 2}}{2+k} \sqrt{\frac{\Lambda_{k}}{k(4+k(1-\sin \rho))}}-\frac{1}{2 \Lambda_{k}} \sqrt{k(4+k(1-\sin \rho))} .
$$

As $\rho \rightarrow \pi / 2$, we have $\Lambda \rightarrow 2$ by (2.4) and

$$
\limsup _{\rho \rightarrow \pi / 2} F\left(\rho, \Lambda_{k}\right) \leq \frac{(2-k)^{3 / 2}}{2+k} \sqrt{\frac{2}{k}}-\frac{\sqrt{k}}{2}
$$

If $k=1$, then

$$
\limsup _{\rho \rightarrow \pi / 2} F\left(\rho, \Lambda_{k}\right) \leq \frac{\sqrt{2}}{3}-\frac{1}{2}<0 .
$$

Together with the fact that $F(\rho, 2)>0$, this shows that there exists $\epsilon>0$ such that

$$
2<\Lambda(\rho)<2+(1-\sin \rho)
$$

for $\rho \in(\pi / 2-\epsilon, \pi / 2)$, which is the second estimate of the lemma.

\subsection{Solution of the second period integral along the graph $\Lambda=\Lambda(\rho)$}

We now show that the second period integral in (2.2),

$$
G(\rho, \Lambda)=\int_{-\frac{\pi}{2}}^{\rho} \frac{\Lambda-4 \sin \rho+2 \sin \phi}{\Lambda-2 \sin \phi} \frac{2-\Lambda \sin \phi}{\Lambda-2 \sin \phi} \frac{d \phi}{\sqrt{\sin \rho-\sin \phi}}
$$

changes sign along $\Lambda=\Lambda(\rho)$. This will guarantee that there exists $\left(\rho_{0}, \Lambda\left(\rho_{0}\right)\right)$ at which both the period conditions (2.1) and (2.2), or, equivalently, (1.8) and (1.9), are satisfied.

According to Lemma 3, it suffices for the discussion of $\rho \rightarrow 0$ to consider $2<\Lambda<8$. The integrand of $G(\rho, \Lambda)$ converges to a positive function as $\rho$ decreases to zero. Therefore $G(\rho, \Lambda)>0$ for $\rho$ near 0 . We will now show that $G(\rho, \Lambda)<0$ for $\rho$ near $\pi / 2$.

As $\rho \rightarrow \pi / 2$, the function $G(\rho, \Lambda)$ may or may not have a limit, depending on how fast $\Lambda(\rho)$ converges to 2 . So we estimate $\lim \sup _{\rho \rightarrow \pi / 2} G(\rho, \Lambda)$ instead, using the second part of the estimates in Lemma 3. 
For each $\rho$ smaller than and sufficiently close to $\pi / 2$, define $\phi_{\rho} \in(0, \rho)$ by the condition

$$
\Lambda-4 \sin \rho+2 \sin \phi_{\rho}=0 .
$$

By Lemma 3, all the terms in the integrand of $G(\rho, \Lambda)$ are positive on $(-\pi / 2, \rho)$, except $\Lambda-4 \sin \rho+2 \sin \phi$, whose sign is the sign of the integrand. This allows us to estimate $G(\rho, \Lambda)$ by two integrals we can control:

$$
G(\rho, \Lambda)=\int_{-\frac{\pi}{2}}^{\phi_{\rho}}+\int_{\phi_{\rho}}^{\rho} \leq \int_{-\frac{\pi}{2}}^{0}+\int_{\phi_{\rho}}^{\rho}
$$

since the integrand of $G(\rho, \Lambda)$ is negative on $\left(-\pi / 2, \phi_{\rho}\right) \supset\left(0, \phi_{\rho}\right)$.

The integral on $(-\pi / 2,0)$ is easy to control. It is continuous at $\rho=\pi / 2$ and $\Lambda=2$, and we have

$$
\begin{aligned}
\lim _{\rho \rightarrow \pi / 2} & \int_{-\frac{\pi}{2}}^{0} \frac{\Lambda-4 \sin \rho+2 \sin \phi}{\Lambda-2 \sin \phi} \frac{2-\Lambda \sin \phi}{\Lambda-2 \sin \phi} \frac{d \phi}{\sqrt{\sin \rho-\sin \phi}} \\
& =-\int_{-\frac{\pi}{2}}^{0} \frac{d \phi}{\sqrt{1-\sin \phi}} \\
& \leq-\int_{-\frac{\pi}{2}}^{0} \frac{d \phi}{\sqrt{1-\phi}}=2(1-\sqrt{1+\pi / 2}) \approx-1.2067 .
\end{aligned}
$$

On the interval $\left(\phi_{\rho}, \rho\right)$, the function $\sin \phi$ is monotonic increasing. It is easy to check that

$$
g(\sin \phi):=\frac{\Lambda-4 \sin \rho+2 \sin \phi}{\Lambda-2 \sin \phi}
$$

is a convex increasing function of $\sin \phi$ with range $[0,1]$. Therefore

$$
g(\sin \phi) \leq g\left(\sin \phi_{\rho}\right)+\frac{g(\sin \rho)-g\left(\sin \phi_{\rho}\right)}{\sin \rho-\sin \phi_{\rho}}\left(\sin \phi-\sin \phi_{\rho}\right) .
$$

That is,

$$
\frac{\Lambda-4 \sin \rho+2 \sin \phi}{\Lambda-2 \sin \phi} \leq \frac{\sin \phi-\sin \phi_{\rho}}{\sin \rho-\sin \phi_{\rho}} .
$$

Since $\Lambda>2$ by (2.4), we have

$$
\frac{2-\Lambda \sin \phi}{\Lambda-2 \sin \phi} \leq 1
$$


so we estimate the integral on $\left(\phi_{\rho}, \rho\right)$ as follows:

$$
\begin{aligned}
\int_{\phi_{\rho}}^{\rho} & \frac{\Lambda-4 \sin \rho+2 \sin \phi}{\Lambda-2 \sin \phi} \frac{2-\Lambda \sin \phi}{\Lambda-2 \sin \phi} \frac{d \phi}{\sqrt{\sin \rho-\sin \phi}} \\
& \leq \frac{1}{\sin \rho-\sin \phi_{\rho}} \int_{\phi_{\rho}}^{\rho} \frac{\sin \phi-\sin \phi_{\rho}}{\sqrt{\sin \rho-\sin \phi}} d \phi \\
& \leq \frac{1}{\sin \rho-\sin \phi_{\rho}} \int_{\phi_{\rho}}^{\rho} \frac{\sin \phi-\sin \phi_{\rho}}{\sqrt{\sin \rho-\sin \phi}} \frac{\cos \phi}{\cos \rho} d \phi \\
& =\frac{1}{\left(\sin \rho-\sin \phi_{\rho}\right) \cos \rho} \cdot \frac{4}{3}\left(\sin \rho-\sin \phi_{\rho}\right)^{3 / 2}=\frac{4}{3} \frac{\sqrt{\sin \rho-\sin \phi_{\rho}}}{\sqrt{1-\sin ^{2} \rho}}
\end{aligned}
$$

Since $\Lambda-4 \sin \rho+2 \sin \phi_{\rho}=0$, by the definition of $\phi_{\rho}$, and $\Lambda \leq 2+(1-\sin \rho)$ by Lemma 3 , we know that

$$
2\left(\sin \rho-\sin \phi_{\rho}\right)=\Lambda-2 \sin \rho \leq 3(1-\sin \rho) .
$$

Therefore

$$
\frac{4}{3} \sqrt{\frac{\sin \rho-\sin \phi_{\rho}}{1-\sin ^{2} \rho}} \leq \frac{4}{3} \sqrt{\frac{\frac{3}{2}(1-\sin \rho)}{1-\sin ^{2} \rho}}=\frac{4 \sqrt{\frac{3}{2}}}{3 \sqrt{1+\sin \rho}}
$$

Thus we have

$$
\limsup _{\rho \rightarrow \pi / 2} \int_{\phi_{\rho}}^{\rho} \frac{\Lambda-4 \sin \rho+2 \sin \phi}{\Lambda-2 \sin \phi} \frac{2-\Lambda \sin \phi}{\Lambda-2 \sin \phi} \frac{d \phi}{\sqrt{\sin \rho-\sin \phi}} \leq \frac{4 \sqrt{\frac{3}{2}}}{3 \sqrt{2}} \approx 1.1547 .
$$

This estimate, together with (2.6), shows that

$$
\limsup _{\rho \rightarrow \pi / 2} G(\rho, \Lambda)<0 .
$$

Therefore $G(\rho, \Lambda)<0$ for $\rho$ near $\phi / 2$, as desired.

\section{Embeddedness}

Theorem 3. Any minimal immersion with Weierstrass data (1.7), which is singly periodic (i.e. satisfies (1.8) and (1.9)), must be an embedding.

We begin by giving a sketch of the proof, which we hope is easy to understand in outline, with references to subsequent sections where the details are carried out. The key idea is to cut a translational fundamental domain of the surface into four 
congruent pieces and then show that each piece is a graph over a domain in the $\left(x_{1}, x_{2}\right)$-plane. The graphs meet only along their embedded boundary arcs.

To produce a translational fundamental domain, we cut the torus on the hdiagonal from one puncture to the other, along the segment of the diagonal that contains the vertex (labelled $O^{\prime}$ in Figure 8). The Weierstrass integral (1.2) is single valued on this slit torus and produces as image a fundamental domain bounded above and below by horizontal straight lines that cross the vertical axis of the surface. These lines are the images of the slit.

We now want to cut up the slit torus into regions whose images are graphs over the $\left(x_{1}, x_{2}\right)$-plane. This means that the cuts must include all points where the Gauss map is horizontal, i.e. all points where $|g|=1$. This set consists of the v-diagonal, together with the line (on the torus) parallel to the h-diagonal and passing through the branch points of $g$ (labelled $b, \hat{b}$ in Figure 8) on the vdiagonal. This decomposes the slit torus into two rectangular pieces. We cut once more along the h-diagonal from end to end along the segment through the center point (labelled $O$ in Figure 8), producing four rectangles.

From Theorem 1i) in Section 1, we know that the images of the four rectangles under the Weierstrass integral $X$ in (1.2) are congruent. On the interior of each rectangle, $|g| \neq 1$, so the projection onto the $\left(x_{1}, x_{2}\right)$-plane of its image under $X$ is an immersion. We will prove in Proposition 1 below that, in fact, the projection is one-to-one, i.e. the image of the interior of each rectangle is a graph. This requires us to control carefully the behavior of the image of the boundaries of the rectangles. These boundaries consist of three different parts (See Figure 9):

- The image of half the h-diagonal. This consists of two parallel horizontal halfrays. One, the image of $H_{1}$, begins at $X(O)$, which we will place at the origin in $R^{3}$. The other, the image of $H_{2}$, begins at $X\left(O^{\prime}\right)$ on the $x_{3}$-axis;

- The image of two segments of the v-diagonal. (These are $E$ and $\hat{E}$, which add up to half of the v-diagonal. Each piece connects $X(b)$ or $X(\hat{b})$ to a point of intersection of the $\mathrm{v}$ - and h-diagonals.) This consists of two disjoint segments on the vertical axis, separated by half a period;

- The image of the remaining arc, on which $|g|=1$ (labelled $C$ in Figure 9). This curve joins the branch points of $g$ on the vertical axis. The third component of the curve is a decreasing function on $C$, which implies that the curve is embedded. Since the arc contains no branch points of $g$ in its interior, it projects to a curve in the $\left(x_{1}, x_{2}\right)$-plane whose tangent vector rotates at a speed that is never zero. This means that the projected loop, $c$, is everywhere locally convex. Since the total turning of its tangent vector is less than $2 \pi$, it is convex.

Putting together the above information, which will be established in Proposition 1, with some more details and arguments, we will show that the boundary of each rectangle is embedded by $X$ and that the interior of each rectangle is em- 
bedded as a graph over a halfplane, minus the interior of the convex loop $c .^{3}$ The curve $c$ has one point in common with the boundary of the halfplane.(See Figure 9, bottom left.)

The proof of Theorem 3 is completed in Section 3.3, where it is shown that the four open graphs are disjoint and that the union of their boundary curves is embedded.

\subsection{The statement of Proposition 1}

As described in the previous section, we cut the torus into four rectangles by removing the points where $|g|=1$, as well as the h-diagonal, along which $g$ is real. The function

$$
g=\frac{w-r e^{i \pi / 4}}{w+r e^{i \pi / 4}}
$$

is unitary precisely on the set of points where $w$ takes values in $e^{-i \pi / 4} \mathbb{R}$, and we know from Section 1.3 that this set consists of the v-diagonal, together with the line orthogonal to this diagonal passing through the branch points of $g$. (Note that this curve passes through the off-axis fixed points of the normal symmetry, which are also branch points of $z$.) Reflection in the diagonals and rotation about $O \in T^{2}$ induce Euclidean motions that act transitively on the four images of these regions under $X$. (See Figures 8 and 9).

Each of the four regions has the property that $|g| \neq 1$ on its interior. In two of them, $|g| \geq 1$, while $|g| \leq 1$ in the other two. We will work with the closed region labeled $\mathcal{D}$ in Figure 8, where $|g|>1$ on the interior, with $|g|=1$ on $E \cup \hat{E} \cup C$, and $g\left(H_{1} \cup H_{2}\right) \subset \mathbb{R}$.

We choose to integrate from $O \in T^{2}$ in the Weierstrass representation (1.2) and we place $X(O)$ at the origin of $\mathbb{R}^{3}$; i.e. $X(O)=\mathbf{O}:=(0,0,0)$. With this normalization, it follows from Theorem 1 that the v-diagonal is mapped into the $x_{3}$-axis. The form of $d h$ in (1.7) and the fact that $z$ takes values of the form it, $t \leq 0$, on this axis (see Figure 3), imply that $x_{3}$ increases monotonically as one descends the $v$-diagonal from 0 to $b$.

The segment of the h-diagonal passing through $O$ and terminating at the punctures must be mapped onto a horizontal line through $X(O)=\mathbf{O}$ in $\mathbb{R}^{3}$. Observing that $g(O)=g\left(O^{\prime}\right)=-1$, while $g=1$ at the other fixed points of the normal symmetry (where $z=\infty$ ), we can conclude that this horizontal line must be the $x_{2}$-axis. Orientation considerations or a direct computation of $x_{2}$ imply that $x_{2}$ is decreasing as one travels from $O$ along $H_{1}$ on the h-diagonal.

\footnotetext{
3 Note that if the normal to a minimal surface is horizontal along a curve that projects to a strictly convex curve, $c$, the minimal surface near the curve projects to the outside of $c$.
} 

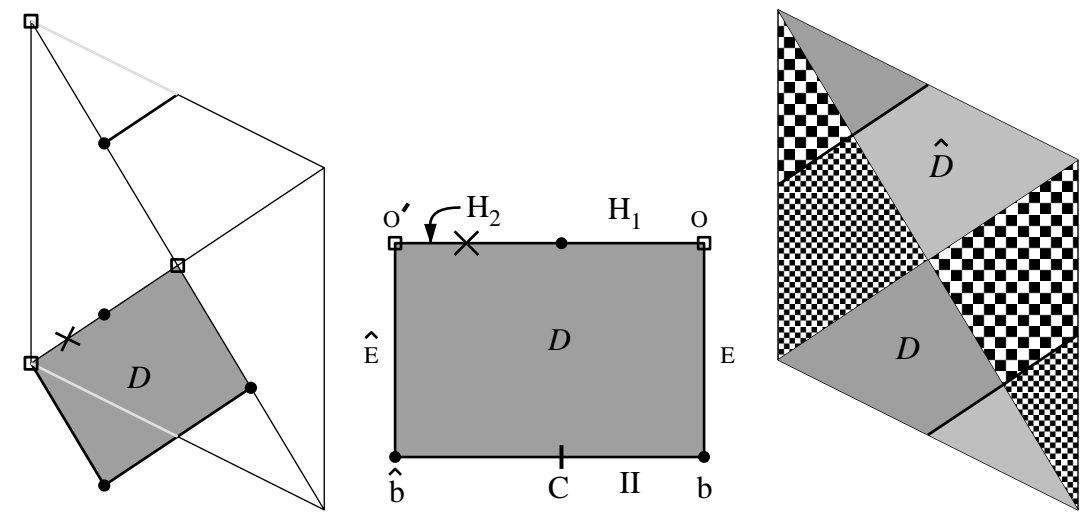

Figure 8.

Left and Center: The region $\mathcal{D}$, whose image is a graph over a halfplane minus a compact convex set. Right: The four regions, each congruent to $\mathcal{D}$ by automorphisms that extend to Euclidean symmetries. The region $\hat{\mathcal{D}}=r_{P} \mathcal{D}$ is referred to in the proof of Theorem 3. The puncture $(\mathrm{x})$ divides the segment $O O^{\prime}$ into the subsegments $H_{1}$ and $H_{2}$.

\section{Proposition 1.}

(i) $X(\mathcal{D})$ is the bounded graph of a function $F$ over an unbounded domain $\Omega$ in the half plane $\left\{\left(x_{1}, x_{2}\right) \mid x_{1} \leq 0\right\}$. The boundary of $\Omega$ consists of the $x_{2}$-axis, together with a compact convex curve $c$, that begins and ends at $(0,0)$ and is symmetric with respect to the $x_{1}$-axis. The function $F$ satisfies $-T / 2 \leq$ $F\left(x_{1}, x_{2}\right) \leq a$, where $T$ is the translational period defined in (1.10), and $a>0$ is defined by $X(b)=(0,0, a)$, where $b$ is the endpoint of $E$ in Figure 8;

(ii) $X(\partial \mathcal{D})$ is an embedding: $X\left(H_{1}\right)$ is the negative $x_{2}$-axis; $X\left(H_{2}\right)$ is the positive $x_{2}$-axis translated by $(0,0,-T / 2) ; X(E)$ is the segment $(0, a)$ on the $x_{3}$-axis; $X(\hat{E})$ is the segment $(-a,-T / 2)$ on the $x_{3}$-axis; $X(C)$ is a monotone graph over the curve $c$ defined in (i) above. It joins $X(b)=(0,0, a)$ to $X(\hat{b})=$ $(0,0,-a)$.

We will prove Proposition 1 in the next section. The reader may wish to read Section 3.3 first, where Theorem 3 is proved using Proposition 1.

\subsection{The proof of Proposition 1}

We will follow the boundary of $\mathcal{D}$, beginning at $O$ and we will show that $\partial \mathcal{D}$ is embedded by $X$ and that - except for the vertical line segments $X(E)$ and $X(\hat{E})$ on the $x_{3}$-axis- $X(\partial \mathcal{D})$ is a graph over a curve in the $\left(x_{1}, x_{2}\right)$-plane. Along the way, we show that points of $\mathcal{D}$ near $\partial \mathcal{D}$ are mapped by $X$ to points that project into $\Omega$, and that points of $\mathcal{D}$ near $\partial \mathcal{D}$ are mapped by $X$ to a graph over a neighborhood 

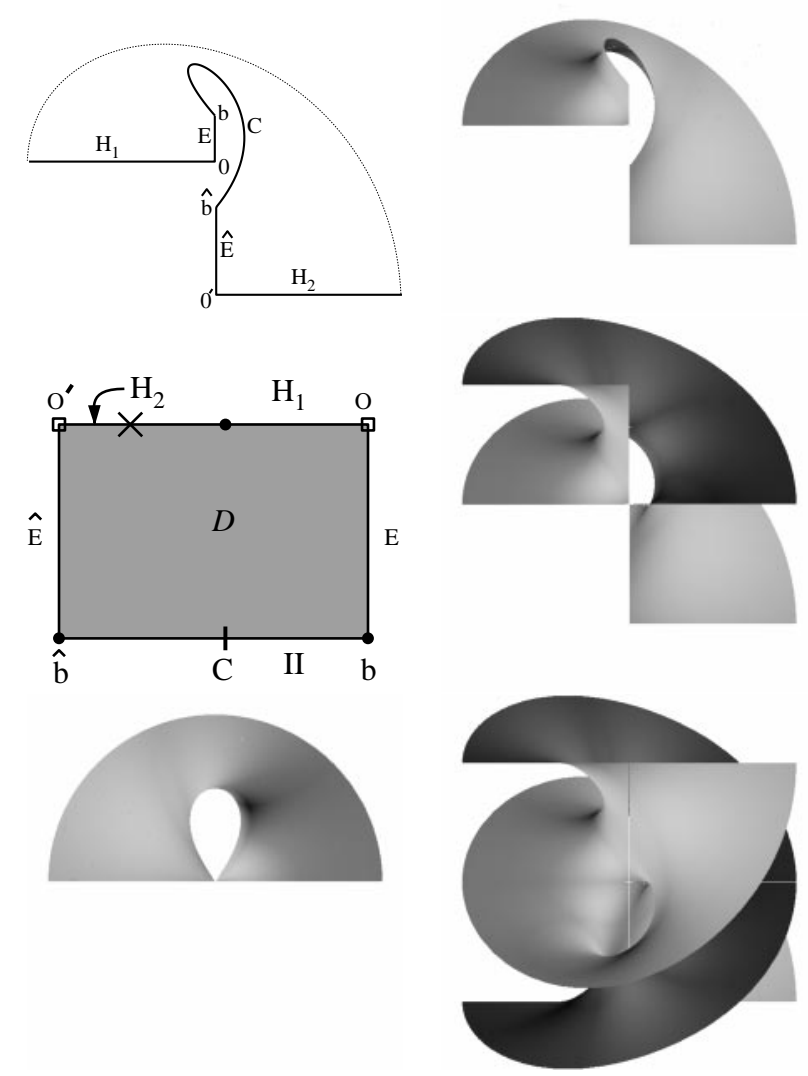

Figure 9 .

Left Column: The domain $\mathcal{D}$ with the image under $X$ of $\partial \mathcal{D}$ above and $\Omega$ the projection of $X(\mathcal{D})$ below. The curves of $X(\partial \mathcal{D})$ are labelled by their preimages in $\partial \mathcal{D}$. Right Column: The graph $X(\mathcal{D})$ at the top, with $X(\mathcal{D}) \cup X(\hat{\mathcal{D}})$ in the middle. Extending the surface by rotation about the vertical axis produces the fundamental domain, bottom right. Note that these surfaces are tilted forward, so $x_{3}(C)$ does not appear to be monotonic, which it actually is.

of $\partial \Omega$ in $\stackrel{\Omega}{ }$. This, together with the behavior of $X$ near the puncture, will allow us to conclude that $X(\mathcal{D})$ is a graph over $\Omega$.

We define

$$
p:=\pi \circ X,
$$

where $\pi$ is the projection of $\mathbb{R}^{3}$ onto the $\left(x_{1}, x_{2}\right)$-plane. Because $|g|>1$ on $\mathcal{D}, p$ is an immersion. Beginning at $O \in T^{2}$, which is mapped to $\mathbf{O} \in \mathbb{R}^{3}$, the v-diagonal is mapped into the $x_{3}$-axis and $x_{3}$ increases monotonically as one descends the 
v-diagonal. ${ }^{4}$ In particular, $p(E)=(0,0)$ and

$$
X(b)=(0,0, a)
$$

for some $a>0$. We may describe the normal symmetry of the surface by $X \circ r_{P}=$ $s \circ X$, where

$$
s\left(x_{1}, x_{2}, x_{3}\right)=\left(x_{1},-x_{2},-x_{3}\right)
$$

Writing $\hat{b}:=r_{P} b$, we have

$$
X(\hat{b})=s \circ X(b)=(0,0,-a) .
$$

We have shown earlier that $X$ maps $H_{1}$, monotonically onto the nonpositive $x_{2}$ axis. Therefore $p=X$ on $H_{1}$.

At $O \in T^{2}, g=-1$. Moreover, $g$ is real and initially decreasing along $H_{1}$; after passing through the vertical point, where $g=\infty, g$ continues to decrease until one reaches a branch point, where $g$ has a value between 1 and $\infty$. (We know from Theorem 1 that $\lambda<1$, so the branch point of $g$ comes before one reaches the puncture. See Figure 6.) Then $g$ increases to $\infty$ as $H_{1}$ diverges to the end. We can deduce two things from this.

First, because $\mathcal{D}$ is to the left of $H_{1}$ as one travels along $H_{1}$ away from $O$, a neighborhood of $H_{1}$ in $\mathcal{D}$ is a graph over a one-sided neighborhood of the negative $x_{2}$-axis, on the side where $x_{1} \leq 0$. Consequently a suitably small neighborhood in $\mathcal{D}$ of the end, which is asymptotic to a helicoidal graph, must be a graph over a region in $\left\{\left(x_{1}, x_{2}\right) \mid x_{1} \leq 0\right\}$.

Second, we can conclude that the surface normal rotates counter-clockwise as one moves away from $O$ along $H_{1}$ and it keeps rotating in this sense until one reaches the branch point of $g$. The two straight lines on the surface, which cross at $\mathbf{O} \in \mathbb{R}^{3}$, are geodesic asymptotic curves. On the conjugate minimal immersion $X^{*}$, defined in a neighborhood of $O \in T^{2}$, the lines correspond to geodesic principal curvature lines, with principal curvatures given by the rate of change of the surface normal along $E$ and $H_{1}$. Since $O \in T^{2}$ is not a branch point of $g$ and $X$ and $X^{*}$ have the same principal curvatures, it follows that the normal to the surface rotates clockwise as one descends $E$ from $O$. Because $\mathcal{D}$ is on the right as one descends $E$ on the torus, it follows that, near $X(E), X(\mathcal{D})$ is a graph over a region in $\left\{\left(x_{1}, x_{2}\right) \mid x_{1}<0\right\}$. (This can also be deduced from computation of the values of $g$ along $E$.) It also implies that, near $X(b)=(0,0, a), X(C-\{b\})$ is a graph over a curve in $\left\{\left(x_{1}, x_{2}\right) \mid x_{1}<0\right\}$.

Along $C, z$ is unitary. On $C, z$ is branched only at the midpoint of $C$, where $z=-e^{-i \rho}$. We may use the phase of $z$ to parameterize $C$ from $b$, where $z=-i$, to the midpoint of $C$, where $z=-i e^{-i(\pi / 2+\rho)}$. That is

$$
C(t):=b+f(t) e^{i \pi / 4}
$$

\footnotetext{
4 See the computation of $d h(\dot{E})$ near the end of Section 1.5 .
} 
is the point where $z \circ C(t)=-i e^{-i t}, 0 \leq t \leq \pi / 2+\rho<\pi$.

From Remark 2 at the end of Section 1.5, $x_{3} \circ I I(t)$ is a strictly decreasing function. (See Figure 7.) But $I I$ is one half of $C$, from $b$ to the midpoint. By using the normal symmetry we can conclude that $x_{3}$ is a decreasing function on all of $C$. In particular $X$ embeds $C$.

Because $g$ is unitary on $C$, the projection

$$
c:=p(C)=\pi \circ X(C)
$$

is a plane curve whose normal vector at $c(t)$ is $g(C(t))$. The Gauss map is branched at $b$ and $\hat{b}$, where $g$ assumes complex conjugate values, but has no branch points on the interior of $C$. Hence $c$, which begins and ends at $(0,0)$, has a normal that turns at a nonzero rate all along $c$, and turns more than $\pi$ but less than $2 \pi$ from beginning to end. ${ }^{5}$ We have already established that near $X(b), X(C)$ projects into the half space $\left\{\left(x_{1}, x_{2}\right) \mid x_{1}<0\right\}$. (See Figure 9.) Hence $c$ is an embedded convex curve in $\left\{\left(x_{1}, x_{2}\right) \mid x_{1} \leq 0\right\}$ that begins and ends at $(0,0)$. It is symmetric with respect to the $x_{1}$-axis: Since $\pi \circ s=\hat{s} \circ \pi$, where $\hat{s}\left(x_{1}, x_{2}\right):=\left(x_{1},-x_{2}\right)$,

$c(t)=p \circ C(t)=\pi \circ X(C(t))=\pi \circ s \circ X(C(-t))=\hat{s} \circ \pi \circ X(C(-t))=\hat{s}(c(-t))$.

We define $\Omega$ to be the unbounded region of $\left\{\left(x_{1}, x_{2}\right) \mid x_{1} \leq 0\right\}$ bounded by the $x_{2}$-axis and $c$. Observe that the normal to the surface along $c$ points out from $\Omega$. Since $c$ is strictly convex and the Gaussian curvature $K$ is never zero along $X(c)$, except at the end points, the projection of any curve on the surface orthogonal to $X(C)$ must lie in $\Omega$ for points sufficiently close to $X(C)$. In particular, $C$ has a neighborhood $\mathcal{U}$ with $p(\mathcal{U}) \subset \Omega$. Because $\mathcal{D}$ is to the right of $C$ as one travels from $b$ toward $\hat{b}, X(\mathcal{U} \cap \mathcal{D})$ is below $X(\stackrel{\circ}{C})$, and $X(\mathcal{U} \cap \mathcal{D})$ is a graph over $p(\mathcal{U} \cap \mathcal{D}) \subset D$.

We can now continue around $\partial \mathcal{D} . X(\hat{E})$ is a segment of the $x_{3}$-axis beginning at $X\left(b_{2}\right)=(0,0,-a)$ and descending to $X\left(O^{\prime}\right)$. Since successive rotation about the horizontal lines generates a translational period, $X\left(O^{\prime}\right)=(0,0,-T / 2)$, where $(0,0, T)$ is the period vector of the singly periodic surface.

Near $X(\hat{b}), X(\mathcal{D})$ projects into $\Omega$. The normal to the surface along $\hat{V}$ turns clockwise as one ascends from $\hat{b}$ to $O^{\prime}$ (descending from $X(\hat{b}) X\left(O^{\prime}\right)$ ) and $g\left(O^{\prime}\right)=$ -1 . Since $\mathcal{D}$ lies to the right of $\hat{E}$ as one ascends, points of $\mathcal{D}$ near $\hat{E}$ are mapped by $p=\pi \circ X$ to points near $(0,0)$ lying in $\left\{\left(x_{1}, x_{2}\right) \mid x_{1}<0\right\}$ and to the right of the

$5180^{\circ}$ rotation around the v-diagonal produces a curve $\tilde{C}$ on $T^{2}$ with $g(\tilde{C})=$ $-g(C)$. Since the degree of $g$ is two, and $g$ is unitary along $C, g(C)$ is an arc of length less than $2 \pi$. Because it begins and ends at $(0,0)$ and has $g=+1$ at its midpoint, $g(C)$ is an arc of length greater than $\pi$. Alternatively, using the fact (established in Theorem 1) that $\rho>0$, we can show that the $g(C)$ is an arc of length less than $2 \pi$. 
tangent line to $c$ at its end point (i.e. to the right of $g(\hat{b})^{\perp}$ ). In particular, near $\hat{E}, \mathcal{D}$ is a graph over a region of $\Omega$.

Arguments similar to those given above easily show that $X\left(H_{2}\right)$ is the ray

$$
\{(O, \tau,-T / 2) \mid \tau>0\},
$$

and $\tau \rightarrow \infty$ as one approaches the puncture at the end of $H_{2}$. We already know that, near the puncture, $X(\mathcal{D})$ is a graph over a region of $\Omega$. We also know this at $\mathrm{O}^{\prime}$, the other end of $H_{2}$. Since $g$ is never unitary on $H_{2}-\left\{O^{\prime}\right\}$, it follows that $H_{2}$ has a neighborhood in $\mathcal{D}$ that is a graph over a neighborhood in $\Omega$ of the positive $x_{2}$-axis.

We now have established statement (ii) as well as the fact that $X(\partial \mathcal{D})$ projects onto $\partial \Omega$. In addition, we have shown that a neighborhood of $\partial \mathcal{D}$ in $\mathcal{D}$, say $\mathcal{R}$, is mapped to a graph over a neighborhood in $\Omega$ of $\partial \Omega$ and that a neighborhood in $\mathcal{D}$ of the puncture is a graph over a region in $\Omega$ of the form $\left\{\left|\left(x_{1}, x_{2}\right)\right|>M\right\}$, for $M$ sufficiently large. This means that $X(\mathcal{R}-\partial \mathcal{D})$ is a graph over a region in $\Omega$ of the form $p(\mathcal{R}) \cup\left\{\left(x_{1}, x_{2}\right) \in \Omega||\left(x_{1}, x_{2}\right) \mid>M\right\}$. The complement of this region in $\Omega$ is compact and simply connected and has a boundary over which $X(\partial \mathcal{R})$ is graph. Since $p$ is an immersion on $\mathcal{D}$, it follows that $X(\mathcal{D}-\mathcal{R})$ is a graph over this complementary region. Hence $X(\mathcal{D})$ is a graph of some function, which we call $F$, over $\Omega$, and is asymptotic to a helicoidal graph as $\left|\left(x_{1}, x_{2}\right)\right| \rightarrow \infty$. In particular, we have proved the first claim of statement (i). Because $\Omega$ lies in the half plane $\left\{\left(x_{1}, x_{2}\right) \mid x_{1} \leq 0\right\}$ and $-T / 2 \leq x_{3} \leq a$ on $X(\partial \mathcal{D})$, it follows that $X(\mathcal{D})$ lies in the slab of $\mathbb{R}^{3}$ defined by these two constraints. This gives the bounds on $F$ stated in statement (i).

\subsection{The proof of Theorem 3}

It suffices to prove that a fundamental piece of the surface, modulo translations, is embedded. We know that such a fundamental piece is made up of four copies of the closure of the graph $X(\mathcal{D})$, described in Proposition 1. Let $\hat{\mathcal{D}}=r_{P} \mathcal{D}$.

Claim. $X$ is an embedding of $\mathcal{D} \cup \hat{\mathcal{D}}$ with values in the slab $\left\{\left(x_{1}, x_{2}, x_{3}\right) \mid x_{1} \leq\right.$ $\left.0,-T / 2 \leq x_{3} \leq T / 2\right\}$ and boundary values consisting of vertical line segments and horizontal rays on $\left\{x_{1}=0\right\}$.

Assuming the claim, which we will prove below, we can complete the proof of Theorem 3.

The rest of a fundamental domain of the surface is produced from $X(\mathcal{D} \cup \hat{\mathcal{D}})$ by $180^{\circ}$ rotation about the $x_{3}$-axis; according to (1.14),

$$
X \circ \mu_{\text {vert }}(p)=\left(-x_{1},-x_{2}, x_{3}\right)(p)=: \sigma \circ X(p)
$$

This means that $\sigma \circ X(\mathcal{D} \cup \hat{\mathcal{D}})$ lies in the slab $\left\{\left(x_{1}, x_{2}, x_{3}\right)\left|x_{1} \geq 0,\right| x_{3} \mid \leq T / 2\right\}$, and is therefore disjoint from $X(\mathcal{D} \cup \hat{\mathcal{D}})$ except along their common boundary, where we already know that $X$ is one-to-one. Hence $X$ is an embedding. 
Proof of the Claim. Recall from (3.1) that $s\left(x_{1}, x_{2}, x_{3}\right)=\left(\hat{s}\left(x_{1}, x_{2}\right),-x_{3}\right)$, where $\hat{s}\left(x_{1}, x_{2}\right):=\left(x_{1},-x_{2}\right)$. Since $X(\hat{\mathcal{D}})=X\left(r_{P} \circ \mathcal{D}\right)=s \circ X(\mathcal{D}), X(\hat{\mathcal{D}})$ is the graph of the function

$$
\hat{F}\left(x_{1}, x_{2}\right):=-F \circ \hat{s}\left(x_{1}, x_{2}\right)
$$

on $\Omega-\{\mathbf{O}\}$, where $F$ is the function, described in Proposition 1, whose graph is $X(\mathcal{D})$. Since $\hat{s}$ interchanges the positive and negative $x_{2}$-axis, on which $F=$ $-T / 2>0$ and $F=0$, respectively, (3.2) implies that $\hat{F}>F$ on these rays.

For $\left|\left(x_{1}, x_{2}\right)\right|$ large, $X(\mathcal{D})$ is asymptotic to the graph of a helicoid over

$$
\left\{\left(x_{1}, x_{2}\right) \mid x_{1} \leq 0,\left(x_{1}, x_{2}\right) \neq(0,0)\right\}
$$

whose boundary values are $-T / 2$ on $\left\{x_{2}>0\right\}$ and 0 on $\left\{x_{2}<0\right\}$. From (3.2) it follows that $\hat{F}>F$ for $\left|\left(x_{1}, x_{2}\right)\right|$ large.

Recall that $c$ is the projection of $X(C)$ onto the $\left(x_{1}, x_{2}\right)$-plane. Because $s \circ$ $X(C)=X(C)$ and $c \circ \hat{s}=c, \hat{F}=F$ on $c$. According to Proposition $1, X$ is one-to-one on $C$. Therefore $X$ is one-to-one on a neighborhood of $C$, so $F \neq \hat{F}$ near, but not on, $c$. The line segments $X\left(H_{1}\right)$ and $X\left(H_{2}\right)$ are disjoint segments of the $x_{3}$-axis and $s \circ X\left(H_{1}\right)$ and $s \circ X\left(H_{2}\right)$ meet $X\left(H_{1}\right) \cup X\left(H_{2}\right)$ only at $X(b)$ and $X(\hat{b})$, near which $X$ is an embedding.

We have shown at each point $\left(x_{1}, x_{2}\right) \in \partial \Omega$ that either $\hat{F}\left(x_{1}, x_{2}\right)>F\left(x_{1}, x_{2}\right)$ or $\hat{F}\left(x_{1}, x_{2}\right)=F\left(x_{1}, x_{2}\right)$ and that $\hat{F}>F$ for nearby points in $\mathcal{D}$. Also, $\hat{F}\left(x_{1}, x_{2}\right)>$ $F\left(x_{1}, x_{2}\right)$ for $\left|\left(x_{1}, x_{2}\right)\right|$ sufficiently large. By the Maximum Principle, $\hat{F}>F$ on $\Omega$ because the set of points in $\AA$ where $\hat{F} \leq F$ is bounded and can have no limit points on $\partial \Omega$. Thus the graphs of $\hat{F}$ and $F$ over $\AA$ are disjoint, which means that $X(\mathcal{D} \cup \hat{\mathcal{D}})$ is an embedding, as claimed. The boundary of $X(\mathcal{D} \cup \hat{\mathcal{D}})$ consists of the two vertical line segments of $X(\mathcal{D})$ and $X(\hat{\mathcal{D}})$, together with the two horizontal rays $\left\{x_{3}=-T / 2, x_{1}=0, x_{2} \geq 0\right\},\left\{x_{3}=T / 2, x_{1}=0, x_{2} \leq 0\right\}$ and the horizontal line $\left\{x_{1}=x_{3}=0\right\}$.

\section{References}

[1] W. Fischer and E. Koch, On 3-periodic minimal surfaces, Zeitschrift für Kristallographie $\mathbf{5 2}$ (1987) 31-52.

[2] W. Fischer and E. Koch, On 3-periodic minimal surfaces with non-cubic symmetry, Zeitschrift für Kristallographie 183 (1988), 129-152.

[3] D. Hoffman and H. Karcher, Complete embedded minimal surfaces of finite total curvature. In E. Osserman (ed.), Encyclopedia of Mathematics, Vol.90, Geometry V. Springer Verlag, 1997, 5-93.

[4] D. Hoffman, H. Karcher and F. Wei, Adding handles to the helicoid, Bulletin of the AMS, New Series 29(1) (1993), 77-84.

[5] D. Hoffman, H. Karcher and F. Wei, The genus one helicoid and the minimal surfaces that led to its discovery. In: K. Uhlenbeck (ed.), Global Analysis and Modern Mathematics, Publish or Perish Press, 1993, pp. 119-170.

[6] W. H. Meeks III and H. Rosenberg, The geometry of periodic minimal surfaces, Comment. Math. Helvetici 68 (1993), 538-578. 
[7] U. Pinkall and K. Polthier, Computing discrete minimal surfaces and their conjugates, Experimental Mathematics 2(1), (1993), 15-36.

David Hoffman

Mathematical Sciences Research Institute

1000 Centennial Drive

Berkeley, CA 94720

USA

e-mail: david@msri.org
Fusheng Wei

Department of Mathematics

Virginia Tech

Blacksburg, VA 24061-0123

USA

e-mail: fwei@calvin.math.vt.edu

Hermann Karcher

Mathematisches Institut Universität Bonn

Beringstrasse 1

D-53115 Bonn

Germany

e-mail: karcher@math.uni-bonn.de

(Received: May 1996; revised October 1996) 